\title{
The Fragmented Glueball: A Personal View
}

\author{
Eberhard Klempt ${ }^{1, *}$ \\ ${ }^{1}$ Helmholtz-Institut für Strahlen- und Kernphysik der Universität Bonn, Nussallee 14-16, 53115 Bonn, \\ Germany
}

Abstract. A coupled-channel analysis has been performed to identify the spectrum of scalar mesons. The data include BESIII data on radiative $J / \psi$ decays into $\pi^{0} \pi^{0}, K_{S} K_{S}, \eta \eta$, and $\omega \phi, 15$ Dalitz plots from $\bar{p} N$ annihilation at rest at LEAR, the CERN-Munich multipoles for $\pi \pi$ elastic scattering, the $S$-wave from BNL data on $\pi \pi$ scattering into $K_{S} K_{S}$, from GAMS data on $\pi \pi \rightarrow \pi^{0} \pi^{0}, \eta \eta$, and $\eta \eta^{\prime}$, and NA48/2 data on low-mass $\pi \pi$ interactions from $K^{ \pm} \rightarrow \pi \pi e^{ \pm} v$ decays. The analysis reveals the existence of ten scalar isoscalar resonances. The resonances can be grouped into two classes: resonances with a large SU(3) singlet component and those with a large octet component. The production of isoscalar resonances with a large octet component should be suppressed in radiative $J / \psi$ decays. However, in a limited mass range centered at $1900 \mathrm{MeV}$, these mesons are produced abundantly. Mainly-singlet scalar resonances are produced over the full mass range but with larger intensity at $1900 \mathrm{MeV}$. The total scalar isoscalar yield in radiative decays into scalar mesons shows a clear peak which is interpreted as the scalar glueball of lowest mass.

\section{Introduction}

Glueballs, bound states of gluons with any constituent quarks, are a firm prediction of QCD. Lattice gauge theories predict the lowest-mass glueball to have scalar quantum numbers and to have a mass of $1710 \pm 50 \pm 80 \mathrm{MeV}$ [1]. Tensor and pseudoscalar glueballs are expected well above $2000 \mathrm{MeV}$. Analytic approximations to QCD find the scalar glueball in the range from 1850 to $1980 \mathrm{MeV}$ [2-4]. Several observed scalar mesons have been proposed to contain a large glueball fraction but no firm conclusions have been reached so far [5-11]. In a recent paper, a partial-wave analysis of data carrying information on scalar mesons was reported [12]. Here, I present these results and give my personal interpretation.

\section{Data and PWA}

Decisive for the interpretation of the glueball as part of the spectrum of scalar mesons are the BESIII data on radiative $J / \psi$ decays. In this process, the primary $c \bar{c}$ converts into one photon and two gluons (see Fig. 1). The gluons interact thus forming a glueball that decays into the observed final-state particles. Figure 2 shows the invariant mass spectra of $\pi^{0} \pi^{0}, K_{s} K_{s}, \eta \eta$, and $\phi \omega$ in a relative $S$ wave produced in radiative $J / \psi$ decays. In parti-

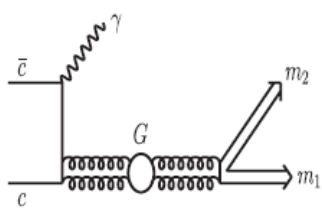

Figure 1. Radiative $J / \psi$ decay

\footnotetext{
*e-mail: klempt@hiskp.uni-bonn.de
} 

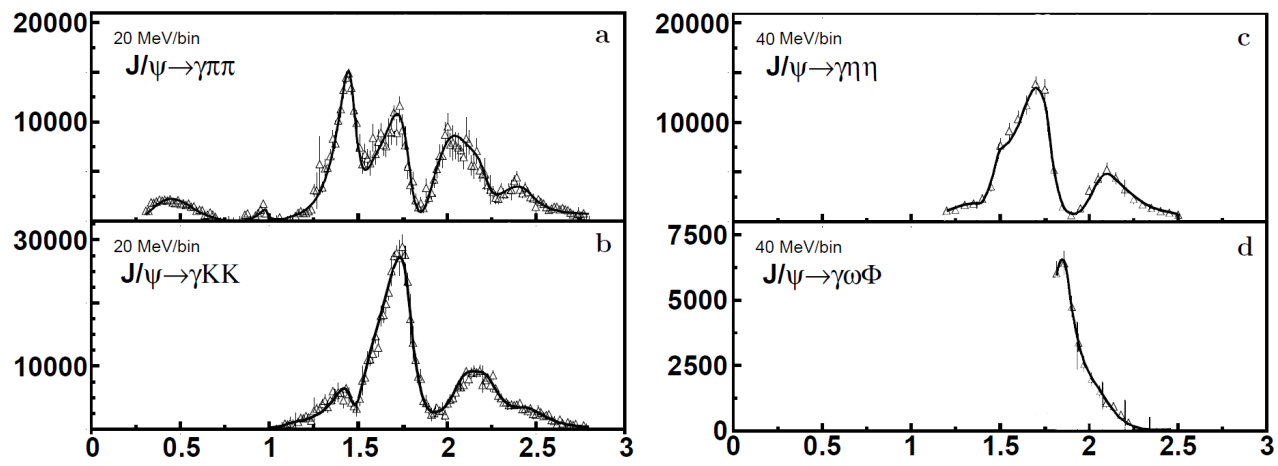

Figure 2. BESIII data on radiative $J / \psi$ data into (a) $\pi^{0} \pi^{0}$, (b) $K_{s} K_{s}$ (b), $\eta \eta$ (c), and $\phi \omega$ (d). Shown is the meson-meson $S$-wave contribution.

cular the $\pi^{0} \pi^{0}$ and $K_{s} K_{s}$ mass distributions show a series of peaks and valleys. These are often seen at different mass positions emphasizing the importance of interference effects and the need of coupled-channel analyses.

These data are important for the interpretation. They are, however, not sufficient to constrain the number and the properties of the contributing resonances. Therefore we added several further data: 15 different Dalitz plots from $\bar{p} N$ annihilation at rest into three pseudoscalar mesons, the multipoles from the CERN-Munich analysis of elastic $\pi \pi$ scattering data - these data allow us to determine the missing intensity of scalar mesons - the GAMS data on $\pi^{+} \pi^{-}$ scattering into $\pi^{0} \pi^{0}, \eta \eta, \eta \eta^{\prime}$, BNL data on $\pi^{+} \pi^{-}$scattering into $K_{s} K_{s}$, and NA48/2 data on low-mass $\pi \pi$ interactions from $K^{ \pm} \rightarrow \pi \pi e^{ \pm} v$ decays.

The analysis finds ten scalar isoscalar resonances. Table 1 shows their masses and widths and compares them to PDG values. The five mesons below $1750 \mathrm{MeV}$ are considered to be established by the Particle Data Group, $f_{0}(1770)$ is "new" (even though evidence had been reported earlier), the four higher-mass mesons were not regarded as being established. The agreement between our new values and those reported earlier is remarkable. Table 2 presents the yields of scalar mesons in the various final states. The missing intensity is derived from the CERN-Munich data and compared to the intensities reported for $J / \psi \rightarrow \gamma 4 \pi$ and $J / \psi \rightarrow \gamma \omega \omega$. The last column gives the sum of all intensity assigned to a specific resonance.

Table 1. Pole masses and widths (in MeV) of scalar mesons. The RPP values are listed as small numbers for comparison.

\begin{tabular}{cccccc}
\hline \hline Name & $f_{0}(500)$ & $f_{0}(1370)$ & $f_{0}(1710)$ & $f_{0}(2020)$ & $f_{0}(2200)$ \\
\hline$M$ & $410 \pm 20$ & $1370 \pm 40$ & $1700 \pm 18$ & $1925 \pm 25$ & $2200 \pm 25$ \\
& $400 \rightarrow 550$ & $1200 \rightarrow 1500$ & $1704 \pm 12$ & $1992 \pm 16$ & $2187 \pm 14$ \\
$\Gamma$ & $480 \pm 30$ & $390 \pm 40$ & $255 \pm 25$ & $320 \pm 35$ & $150 \pm 30$ \\
& $400 \rightarrow 700$ & $100 \rightarrow 500$ & $123 \pm 18$ & $442 \pm 60$ & $\sim 200$ \\
\hline \hline Name & $f_{0}(980)$ & $f_{0}(1500)$ & $f_{0}(1770)$ & $f_{0}(2100)$ & $f_{0}(2330)$ \\
\hline$M$ & $1014 \pm 8$ & $1483 \pm 15$ & $1765 \pm 15$ & $2075 \pm 20$ & $2340 \pm 20$ \\
& $990 \pm 20$ & $1506 \pm 6$ & & $2086_{-24}^{+20}$ & $\sim 2330$ \\
$\Gamma$ & $71 \pm 10$ & $116 \pm 12$ & $180 \pm 20$ & $260 \pm 25$ & $165 \pm 25$ \\
& $10 \rightarrow 100$ & $112 \pm 9$ & & $284_{-32}^{+60}$ & $250 \pm 20$ \\
\hline \hline
\end{tabular}


Table 2. Yields of scalar mesons in radiative $J / \psi$ decays (in units of $10^{-5}$ ). The RPP values are listed as small numbers for comparison.

\begin{tabular}{|c|c|c|c|c|c|c|c|c|}
\hline \multirow[t]{2}{*}{$B R_{J / \psi \rightarrow \gamma f_{0}} \rightarrow$} & \multirow[t]{2}{*}{$\gamma \pi \pi$} & \multirow[t]{2}{*}{$\gamma K \bar{K}$} & \multirow[t]{2}{*}{$\gamma \eta \eta$} & \multirow[t]{2}{*}{$\gamma \eta \eta^{\prime}$} & \multirow[t]{2}{*}{$\gamma \omega \phi$} & \multicolumn{2}{|c|}{ missing } & \multirow[t]{2}{*}{ total } \\
\hline & & & & & & $\gamma 4 \pi$ & $\gamma \omega \omega$ & \\
\hline$f_{0}(500)$ & $105 \pm 20$ & $5 \pm 5$ & $4 \pm 3$ & $\sim 0$ & $\sim 0$ & $\sim$ & & $114 \pm 21$ \\
\hline$f_{0}(980)$ & $1.3 \pm 0.2$ & $0.8 \pm 0.3$ & $\sim 0$ & $\sim 0$ & $\sim 0$ & $\sim$ & & $2.1 \pm 0.4$ \\
\hline \multirow[t]{2}{*}{$f_{0}(1370)$} & $38 \pm 10$ & $13 \pm 4$ & $3.5 \pm 1$ & $0.9 \pm 0.3$ & $\sim 0$ & 14 & & $69 \pm 12$ \\
\hline & & $42 \pm 15$ & & & & $27 \pm 9$ & & \\
\hline \multirow[t]{2}{*}{$f_{0}(1500)$} & $9.0 \pm 1.7$ & $3 \pm 1$ & $1.1 \pm 0.4$ & $1.2 \pm 0.5$ & $\sim 0$ & \multirow{2}{*}{\multicolumn{2}{|c|}{$\begin{array}{l}33 \pm 8 \\
36 \pm 9\end{array}$}} & $47 \pm 9$ \\
\hline & $10.9 \pm 2.4$ & $2.9 \pm 1.2$ & $1.7_{-1.4}^{+0.6}$ & $6.4_{-2.2}^{+1.0}$ & & & & \\
\hline$f_{0}(1710)$ & $6 \pm 2$ & $23 \pm 8$ & $12 \pm 4$ & $6.5 \pm 2.5$ & $1 \pm 1$ & \multicolumn{2}{|c|}{$7 \pm 3$} & $56 \pm 10$ \\
\hline$f_{0}(1770)$ & $24 \pm 8$ & $60 \pm 20$ & $7 \pm 1$ & $2.5 \pm 1.1$ & $22 \pm 4$ & \multicolumn{2}{|c|}{$65 \pm 15$} & $181 \pm 26$ \\
\hline$f_{0}(1750)$ & $38 \pm 5$ & $99_{-6}^{+10}$ & $24_{-7}^{+12}$ & & $25 \pm 6$ & $97 \pm 18$ & $31 \pm 10$ & \\
\hline$f_{0}(2020)$ & $42 \pm 10$ & $55 \pm 25$ & $10 \pm 10$ & & & \multicolumn{2}{|c|}{$(38 \pm 13)$} & $145 \pm 32$ \\
\hline$f_{0}(2100)$ & $20 \pm 8$ & $32 \pm 20$ & $18 \pm 15$ & & & \multicolumn{2}{|c|}{$(38 \pm 13)$} & $108 \pm 25$ \\
\hline$f_{0}(2200)$ & $5 \pm 2$ & $5 \pm 5$ & $0.7 \pm 0.4$ & & & \multicolumn{2}{|c|}{$(38 \pm 13)$} & $49 \pm 17$ \\
\hline$f_{0}(2100) / f_{0}(2200)$ & $62 \pm 10$ & $109_{-19}^{+8}$ & $11.0_{-3.0}^{+6.5}$ & & & \multicolumn{2}{|l|}{$115 \pm 41$} & \\
\hline \multirow[t]{2}{*}{$f_{0}(2330)$} & $4 \pm 2$ & $2.5 \pm 0.5$ & $1.5 \pm 0.4$ & & & & & $8 \pm 3$ \\
\hline & & $20 \pm 3$ & & & & & & \\
\hline
\end{tabular}

The sum of the yields of $f_{0}(1710)$ and $f_{0}(1770)$ should be compared to the RPP entry for $f_{0}(1750)$. RPP reports yields for $f_{0}(2100)$ and $f_{0}(2200)$. The yields can be compared to the sum of three resonances found by us. Above $1900 \mathrm{MeV}$, no data on $\pi \pi$ elastic scattering are known. The total $\gamma 4 \pi$ intensity is therefore distributed between the three states observed in the 2000 to $2200 \mathrm{MeV}$ range.

\section{Interpretation}

The mesons in Table 1 are grouped pairwise. There are two mesons close in mass followed by a mass gap. Figure 3 shows the squared mass values of the "higher-mass" and the "lower-mass" mesons as a function of a consequtive number. The squared masses depend about linearly on the consecutive number. The numbering starts with (-1) since we do not want to imply that $f_{0}(500)$ is the ground state of the scalar-isoscalar-meson spectrum. The "lower" and "higher"-mass mesons are separated by the mass-square difference between $\eta$ and $\eta^{\prime}$ mesons

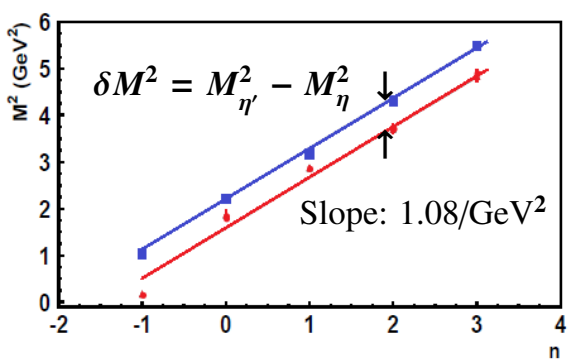

Figure 3. Squared masses of mainly-octet and mainly-singlet scalar isoscalar mesons as functions of a consecutive number. but the spectrum is inverted. This is expected for instanton-induced interactions [17]. For scalar mesons, $f_{0}(500)$ is mainly singlet, $f_{0}(980)$ is mainly octet $[18,19]$. The decay pattern identifies $f_{0}(1370)$ as mainly singlet, $f_{0}(1500)$ as mainly octet state. Thus we conjecture that the $q \bar{q}$ component of the "higher-mass" mesons is mainly in the octet, the "lower-mass" mesons in the singlet configuration. 
Mainly-octet mesons should not be produced in radiative $J / \psi$ decays but they are. Figure 4 shows the total yield of scalar isoscalar mesons in radiative $J / \psi$ decays (from Table 2 ) as a function of the invariant mass. Open circles represent the yield of "highermass", full squares of "lower-mass" scalar mesons. Even though the quark content of the "higher-mass" scalar mesons is supposed to be mainly in the octet configuration, their is significant production in the mass range from 1500 to $2300 \mathrm{MeV}$. This high yield re-

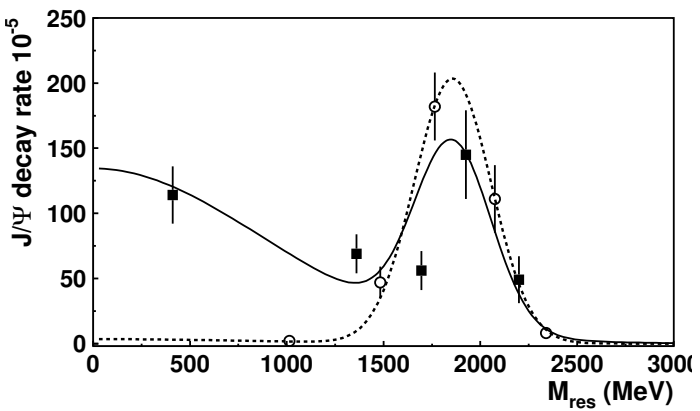

Figure 4. Squared masses of mainly-octet and mainly-singlet scalar isoscalar mesons as functions of a consecutive number. quires a significant singlet contribution in their wave functions. No selection rule forbids SU(3) singlet scalar mesons to be produced in radiative $J / \psi$ decays, they are produced in the full mass range but their production is enhanced in the same mass range. We interpret this enhancement as scalar glueball.
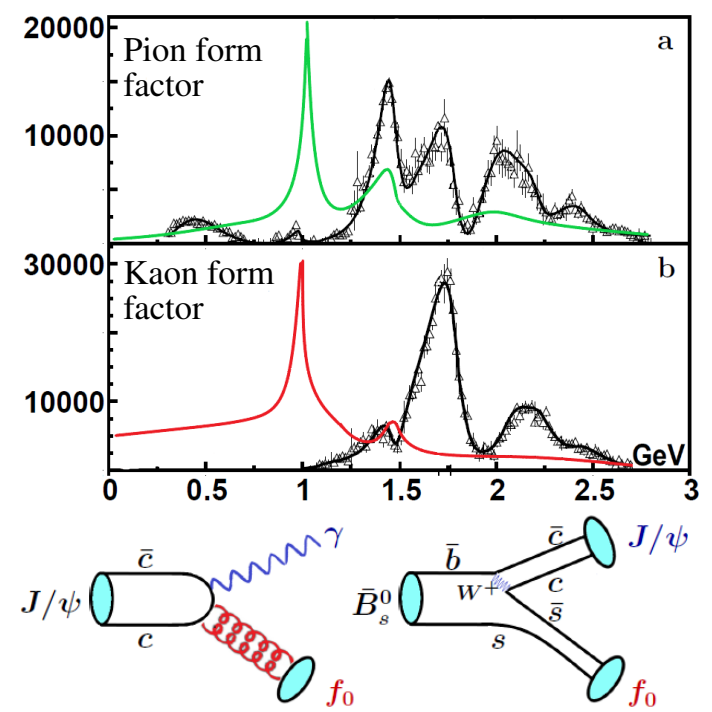

Figure 5. Comparison of scalar-meson production in radiative $J / \psi$ decay and in $\bar{B}_{s}^{0} \rightarrow J / \psi f_{0}$.
This interpretation is confirmed when data on $J / \psi \rightarrow \gamma f_{0}$ and the $\mathrm{LHCb}$ data $\bar{B}_{s}^{0} \rightarrow J / \psi f_{0}[20,21]$ are compared. In $J / \psi$ decays, two gluons convert into the scalar meson that decays into the final-state particles. A montainous landscape is generated with high peaks and deep valleys. For $\bar{B}_{s}^{0}$ decays, the form factors from [22] are shown in Fig. 5. Their square is proportional to the cross section. Very little intensity is observed. This is particulary striking when two kaons are seen in the final state (the kaon form factor). In $\bar{B}_{s}^{0}$ decays an $s \bar{s}$ pair is generated in the initial state but no resonance is produced even though there is a strong $K \bar{K}$ peak when two gluons are in the initial state. The two montains in the $K \bar{K}$ invariant mass at 1700 and $2200 \mathrm{MeV}$ are not generated - or at most weakly - by

$s \bar{s}$ pairs but abundantly in gluon-gluon interactions.

Radiative $J / \psi$ decays produce scalar isoscalar mesons abundantly. Those mesons that are supposed to have a mostly-octet $q \bar{q}$ structure (and that should not be produced in radiative $J / \psi$ decays) are produced but only in a limited mass range, with a clear peak at about $1900 \mathrm{MeV}$. Mostly-singlet isoscalar mesons are produced in the full mass range but the yield is enhanced at about $1900 \mathrm{MeV}$. This peak is not seen in $\bar{B}_{s}^{0}$ decays, it is seen only with two gluons in the initial state. The enhancement can be fit with a Breit-Wigner resonance. The fit returns

$$
\begin{gathered}
M_{\text {glueball }}=(1865 \pm 25) \mathrm{MeV}, \Gamma_{\text {glueball }}=\left(370 \pm 50_{-20}^{+30}\right) \mathrm{MeV} \\
\mathrm{Y}_{\mathrm{J} / \psi \rightarrow \gamma \mathrm{G}_{0}}=(5.8 \pm 1.0) \cdot 10^{-3}
\end{gathered}
$$

This is the largest yield in radiative $J / \psi$ decays into a meson. It is the expected scalar glueball. 


\section{Discussion}

Is this really the expected scalar glueball? At least one aspect is completely unexpected. There are ten scalar isoscalar resonances all falling on a linear $\left(n, M^{2}\right)$ trajectory. All find a spectroscopic identification within the quark model - except perhaps $f_{0}(500)$ and $f_{0}(980)$. There is no extra state, no intruder that enters the spectrum, mixes with other states and increases the number of observed states by one. In Fig. 3, there is no additional resonance. How can that be? The scalar glueball seems to make up a fraction of the wave function of scalar isoscalar mesons without increasing the number of states.

We can write down the quark decomposition of the wave function of a mainly-octet scalar meson:

$$
\begin{aligned}
f_{0} & =\alpha_{1} \frac{1}{\sqrt{6}}(u \bar{u}+d \bar{d}-2 s \bar{s})+\alpha_{2} \frac{1}{\sqrt{6}}(u \bar{u} s \bar{s}+d \bar{d} s \bar{s}-2 u \bar{u} d \bar{d}) \\
& +\alpha_{3} \cdot(\text { meson }- \text { meson cloud })+\alpha_{4}(\mathrm{gg})+\alpha_{5}(\mathrm{q} \overline{\mathrm{q} g}) \\
& + \text { and some singlet contribution }:\left\{\alpha^{\prime} \frac{1}{\sqrt{3}}(\mathrm{u} \overline{\mathrm{u}}+\mathrm{d} \overline{\mathrm{d}}+\mathrm{s} \overline{\mathrm{s}})+\beta^{\prime} \frac{1}{\sqrt{3}}(\mathrm{uu} \overline{\mathrm{s}} \overline{\mathrm{s}}+\mathrm{d} \overline{\mathrm{d}} \mathrm{s} \overline{\mathrm{s}}+\mathrm{uu} \mathrm{d} \overline{\mathrm{d}})\right\}
\end{aligned}
$$

These are five different octet contributions, five Fock components. These could all be realized independently. They could mix, but the number of ground states - and the number of states in every excitation level - could be five. This seems not to be the case. The number of states is rather one per excitation level and not five. The large number of different Fock components does obviously not lead to a large number of states but only to components in the wave functions.

\section{Summary}

The BESIII collaboration reported data on radiative $J / \psi$ decays with unprecedented statistics. The data reveal high intensities in the yield of scalar mesons. The data can be fit with ten scalar isoscalar resonances. The scalar resonances can be grouped into a class of mainlysinglet and mainly-octet states. The two groups fall onto linear $\left(n, M^{2}\right)$-trajectories. Octet scalar isoscalar resonances are produced mainly in the $1700-2100 \mathrm{MeV}$ mass range. Singlet scalar resonances are produced over the full mass range but their intensity peaks in the 1700 - $2100 \mathrm{MeV}$ mass range. The enhanced production of scalar mesons in the $1700-2100 \mathrm{MeV}$ mass range is due to gluon-gluon in the initial state. The peak is the scalar glueball of lowest mass. The glueball does not intrude the spectrum of scalar states as additional resonance. It contributes to the wave function of several scalar isoscalar resonances.

\section{References}

[1] Y. Chen et al., Phys. Rev. D 73, 014516 (2006).

[2] A. P. Szczepaniak and E. S. Swanson, Phys. Lett. B 577, 61-66 (2003).

[3] M. Q. Huber, C. S. Fischer and H. Sanchis-Alepuz, Eur. Phys. J. C 80 no.11, 1077 (2020).

[4] M. Rinaldi and V. Vento, Phys. Rev. D 104, no.3, 034016 (2021).

[5] F. E. Close and A. Kirk, Eur. Phys. J. C 21, 531-543 (2001).

[6] E. Klempt and A. Zaitsev, Phys. Rept. 454, 1 (2007).

[7] V. Mathieu, N. Kochelev and V. Vento, Int. J. Mod. Phys. E 18, 1 (2009).

[8] V. Crede and C. A. Meyer, Prog. Part. Nucl. Phys. 63, 74 (2009).

[9] C. Amsler et al., "Scalar Mesons below $2 \mathrm{GeV}$ ", in: P. A. Zyla et al. [Particle Data Group], PTEP 2020, no.8, 083C01 (2020). 
[10] W. Ochs, J. Phys. G 40, 043001 (2013).

[11] F. J. Llanes-Estrada, Eur. Phys. J. ST 230, no.6, 1575-1592 (2021).

[12] A. V. Sarantsev et al., Phys. Lett. B 816, 136227 (2021).

[13] M. Ablikim et al. [BESIII Collaboration], Phys. Rev. D 92 no.5, 052003 (2015).

[14] M. Ablikim et al. [BESIII Collaboration], Phys. Rev. D 98 no.7, 072003 (2018).

[15] M. Ablikim et al. [BESIII Collaboration], Phys. Rev. D 87, no. 9, 092009 (2013).

[16] M. Ablikim et al. [[BESIII Collaboration], Phys. Rev. D 87 no.3, 032008 (2013).

[17] E. Klempt, B.C. Metsch, C.R. Münz and H.R. Petry, Phys. Lett. B 361, 160-166 (1995).

[18] J. A. Oller, Nucl. Phys. A 727, 353-369 (2003).

[19] E. Klempt, Phys. Lett. B 820, 136512 (2021).

[20] R. Aaij et al. [LHCb], Phys. Rev. D 89, no.9, 092006 (2014).

[21] R. Aaij et al. [LHCb], JHEP 08, 037 (2017).

[22] S. Ropertz, C. Hanhart and B. Kubis, Eur. Phys. J. C 78, no.12, 1000 (2018). 